\title{
Visceral Crisis Means Short Survival Among Patients With Luminal A Metastatic Breast Cancer: A Retrospective Cohort Study
}

\author{
Yassir Sbittia, e, Khaoula Slimani ${ }^{a}$, Adil Debbagha ${ }^{\mathrm{a}}$, Anouar Mokhlis ${ }^{\mathrm{a}}$, Habiba Kadiri ${ }^{\mathrm{b}}$, \\ Abdelilah Laraquic, Hassan Errihani ${ }^{\mathrm{d}}$, Mohamed Ichou ${ }^{\mathrm{a}}$
}

\begin{abstract}
Background: Patients with visceral crisis from luminal metastatic breast cancer $(\mathrm{mBC})$ are often treated with palliative chemotherapy. No studies have analyzed the aggressiveness of the care in visceral crisis from luminal $\mathrm{mBC}$ patients. The objective of this study was to assess practices in this setting in a university medical oncology department.
\end{abstract}

Methods: This retrospective study included all patients who were managed for luminal mBC between January 2013 and April 2016. The analysis focused on the characteristics of the patients, the modalities of cancer treatment and delays between visceral crisis and death.

Results: Thirty-five patients pre-treated with two hormonal therapy lines were enrolled retrospectively. Worse performance status and a higher proportion of severe organ dysfunction for luminal $\mathrm{mBC}$ were observed among patients with visceral crisis. Sixty-five percent of patients received cytotoxic treatment. One cycle of chemotherapy was administrated in the majority of patients. Palliative care was performed in $35 \%$ of patients. Chemotherapy did not have any significant effect on patient outcome in the present study. The mean time between visceral crisis and death was 4.7 weeks (standard deviation $=1.9$ ).

Conclusion: Our study showed that visceral crisis in patients with luminal $\mathrm{mBC}$ is a complex problem. We need more comprehension of molecular pathogenesis to visceral crisis disease to propose effi-

Manuscript submitted May 25, 2017, accepted June 13, 2017

a Department of Medical Oncology, University Military Hospital, Rabat 10000, Morocco

bDepartment of Pathology, National Institute of Oncology, Rabat 10000, Morocco

${ }^{\mathrm{c}}$ Department of Biosecurity, University Military Hospital, Rabat 10000, Morocco

${ }^{\mathrm{d}}$ Department of Medical Oncology, National Institute of Oncology, Rabat 10000, Morocco

${ }^{e}$ Corresponding Author: Yassir Sbitti, Department of Medical Oncology, Rabat Teaching University Military Hospital and Faculty of Medicine and Pharmacy of Fez, Hay Ryad, Rabat 10000, Morocco. Email: sbittiyassir@yahoo.fr

doi: https://doi.org/10.14740/wjon1043w cacious treatments for these patients and to identify subgroup of patients who need chemotherapy followed by maintenance endocrine therapy.

Keywords: Visceral crisis; Metastatic breast cancer; Luminal A; Chemotherapy

\section{Introduction}

Selecting a systemic treatment for metastatic breast cancer $(\mathrm{mBC})$ is a complex process. Systemic treatments prolong survival and enhance quality of life but are not curative; therefore, the use of minimally toxic endocrine therapies is preferred to the use of cytotoxic therapy whenever reasonable. Approximately $67-70 \%$ of all metastatic breast tumors contain estrogen receptor (ER) and progesterone hormone receptor (PHR)-positive cells $[1,2]$. International guidelines recommend hormonal therapy rather than chemotherapy for luminal $\mathrm{mBC}$ without symptomatic visceral disease [3-6]. Endocrine therapy is an important systemic treatment option for pre- and postmenopausal women with advanced breast cancer due to a less toxic side effect profile and comparable efficacy to chemotherapy $[7,8]$. There are a number of endocrine agents that are currently available for the treatment of breast cancer. The choice of agent should be based on the previous agents used, the safety and tolerability of the agent, patient comorbidities, and menopausal status and socioeconomic factors. Recently, a number of novel targeted therapies for the treatment of hormone receptor positive $\left(\mathrm{HR}^{+}\right)$/human epidermal growth factor receptor 2 negative $\left(\mathrm{HER}^{2-}\right) \mathrm{mBC}$ have been approved or are in late-stage clinical development, including mammalian target of rapamycin (mTOR) inhibitors (e.g., everolimus), and cyclin-dependent kinase (CDK)-4/6 inhibitors (e.g., palbociclib and ribociclib). Large phase III randomized controlled trials have demonstrated the superior efficacy of these treatments compared to conventional endocrine monotherapies [9-12]. In visceral crisis, current guidelines recommend chemotherapy to achieve rapid symptom control. No studies have analyzed the aggressiveness of the care in visceral crisis from luminal $\mathrm{mBC}$ patients. This retrospective study aimed to see how chemotherapy decision influenced clinical outcomes including 
Table 1. Characteristics of the Population With Advanced Breast Cancer and Visceral Crisis Disease

\begin{tabular}{ll}
\hline Baseline characteristics & 35
\end{tabular}

Age at breast cancer diagnosis, median years (range)

Educational level, n (\%)

Illiterate

Elementary

Junior college

High school

Marital status, n (\%)

Married

Widow

Menopausal status at the first breast cancer diagnosis, $\mathrm{n}(\%)$

Postmenopausal status, N (\%)

Pre-perimenopausal status, N (\%)

Insurance plan type at $\mathrm{mBC}$ diagnosis, $\mathrm{n}(\%)$

Financial dependence, $\mathrm{n}(\%)$

Partner

Own work

mBC type, n (\%)

De novo

ECOG PS at visceral crisis, $\mathrm{n}(\%)$

2

3

Number of metastatic sites at index treatment initiation

$$
1
$$

2

Sites of metastatic disease at mBC diagnosis, $\mathrm{n}(\%)$

\section{Bone}

Liver

Lung

Positive ER tumor status (70-100\%)

Positive PgR tumor status (70-100\%)

Negative HER2 neu tumor status

Type of index treatment for $\mathrm{mBC}, \mathrm{n}(\%)$

Ovarian suppression

Tamoxifene

Aromatases inhibitor

Fulvestrant

Line of hormonal therapy for $\mathrm{mBC}, \mathrm{n}(\%)$

First line

Second line

Third line

Duration from initiation hormonal treatment to visceral crisis, median months (range)

\section{5}

$48 \pm 8.7$ years

$10(28.57 \%)$

$15(42.85 \%)$

$8(22.85 \%)$

$2(5.71 \%)$

$27(77.14 \%)$

$8(22.85 \%)$

$28(80 \%)$

$7(20 \%)$

$35(100 \%)$

$31(88.57 \%)$

$4(11.42 \%)$

$35(100 \%)$

$31(88.57 \%)$

$4(11.42 \%)$

$19(54.28 \%)$

$12(34.28 \%)$

$4(11.42 \%)$

$15(42.85 \%)$

$27(77.14 \%)$

$14(40 \%)$

$35(100 \%)$

$35(100 \%)$

$35(100 \%)$

$7(20 \%)$

$35(100 \%)$

$35(100 \%)$

$3(8.5 \%)$

$35(100 \%)$

$35(100 \%)$

$25(100 \%)$

$18 \pm 4.3$ months 
survival in luminal $\mathrm{mBC}$ patients with visceral crisis and the main question faced by physician is how to treat or propose palliative care for this patient population.

\section{Materials and Methods}

This study was retrospectively designed. The institutional ethic review board of military teaching university hospital approved the current study. Permission from the hospital authority was taken to access the medical records.

\section{Participants and data collection}

Dataset from the military teaching university hospital medical oncology department tumor database was retrospectively reviewed to identify patients with breast cancer. We included those patients identified to have luminal metastatic disease within visceral crisis. Patients diagnosed between January 2013 and April 2016 were chosen for our study population. Demographic and clinical characteristics of the patients were obtained from the hospital registration records. In context of $\mathrm{mBC}$, visceral crisis is defined as presence of: carcinomatous meningitis or leptomeningeal metastases, and significant liver metastases: usually when causing liver functional compromise - hepatocellular failure - raised bilirubin in absence of extrahepatic obstruction, significantly elevated transaminases with diffuse liver mets, lymphangitic lung metastases, or bulky lung metastases, respiratory failure, and bone marrow replacement. Data concerning the management plan, primary malignancy, stage, and timing of chemotherapy in visceral crisis were also obtained. At the time of diagnosis of "visceral crisis", patients generally had a complete physical examination and ancillary labs that typically included basic blood work (complete cell count and complete blood chemistries including calcium and liver and renal function tests) and serum tumor markers such as cancer antigen (CA) 15-3. Bone scans and computed tomography (CT) scans of biopsies of metastatic sites were performed when feasible. Cytotoxic chemotherapy was considered for all patients depending on their expectations, or a physician decision. Chemotherapy-based treatment was as follows: paclitaxel and bevacizumab, doxorubicin plus cyclophamide, docetaxel, or zoledronic acid administrated in patients with bone mets. Overall survival was calculated to assess the impact of chemotherapy on clinical outcomes.

\section{Statistical analysis}

A retrospective review of clinical and treatment data for all the patients was carried out, and data were entered on an anonymized database for data collection. Our clinical data were expressed in mean and standard deviation (SD), median, or percentage. Overall survival (OS) was calculated by the Kaplan-Meier product-limit method. OS was calculated as the time from the date of visceral crisis to the date of death or last contact. SPSS 20.0 software was used for statistical analysis.
Table 2. Characteristics of the Hospitalizations of Patients With Advanced Breast Cancer and Visceral Crisis Disease

\begin{tabular}{lll}
\hline Causes of death & Effect & Percentage \\
\hline Progression of disease & 17 patients & $48.57 \%$ \\
Hepatic insufficiency & Nine patients & $25.71 \%$ \\
Respiratory failure & Seven patients & $20 \%$ \\
Septic shock & Two patients & $5.71 \%$ \\
\hline
\end{tabular}

\section{Results}

From January 2013 and April 2016, 315 patients with luminal A mBC were treated in Medical Oncology Department from Teaching Military Hospital Mohamed V Morocco. Thirty-five patients with $\mathrm{ER}^{+} / \mathrm{HER}^{2-}$ advanced breast cancer with visceral crisis disease were enrolled in this retrospective study. The study population comprised all patients who had received two hormonal therapy lines. Main patient and tumor characteristics are reported in Table 1. Median age was 48 years (SD 8.7), and median Eastern Cooperative Oncology Group (ECOG) performance status (PS) was 2. According to histopathology, there were 32 patients $(91.4 \%)$ diagnosed as invasive ductal carcinoma, and three $(8.6 \%)$ as invasive lobular carcinoma. All (100\%) patients had de novo $\mathrm{mBC}$ with ER and/or progesterone receptor ( $\mathrm{PgR}$ ) positive primary tumors and HER ${ }^{2-}$. The majority of the enrolled patients had visceral disease $(60.5 \%)$, more than $54 \%$ of the patients had single metastasis and $10 \%$ of the patients had multiple metastatic sites, with a median number of two sites. All $(100 \%)$ of the patients had received endocrine treatment for advanced disease, with a median number of two lines. Endocrine therapy includes ovarian suppression in seven patients $(20 \%)$, tamoxifene in 35 patients $(100 \%)$, aromatase inhibitor in 35 patients $(100 \%)$ and fulvestrant in three patients $(8.5 \%)$. Median duration of hormonal treatment was 18 months $(\mathrm{SD}=4.3)$. Visceral crisis was distributed as follows: carcinomatous meningitis or leptomeningeal metastases in $20 \%$, significant liver metastases causing liver functional compromise - hepatocellular failure - raised bilirubin in absence of extrahepatic obstruction, elevated transaminases in 55\%, lymphangitic lung metastasis, or bulky lung metastases causes' respiratory failure in $35 \%$. In regards to chemotherapy for visceral crisis in advanced disease, $20 \%$ of the patients had been treated with paclitaxel and bevacizumab, $20 \%$ of the patients had received docetaxel, and $25 \%$ of the patients had treated with anthracyclines regimens as treatment for advanced disease, respectively. First cycle of chemotherapy was administrated in the majority of patients $(91 \%)$. Palliative care was performed in $34(30 \%)$ patients (Table 2). Progression of disease was the most common cause of death in 17 patients $(48.57 \%)$ followed by septic shock in two patients $(5.71 \%)$, respiratory failure in seven patients $(20 \%)$ and hepatic insufficiency in nine patients (25.71\%). During the 5 weeks periods preceding death, $65 \%$ of the patients received active treatments (Table 3 ). The mean time between visceral crisis and death was 4.7 weeks $(\mathrm{SD}=$ 2.9). Overall, luminal $\mathrm{mBC}$ patients with visceral crisis had significantly shorter OS. Using chemotherapy as index ther- 
Table 3. Characteristics of the Administered Treatment in Luminal Metastatic Breast Cancer With Visceral Crisis

\begin{tabular}{lll}
\hline & Effect & Percentage \\
\hline Best supportive care & 12 & $34.28 \%$ \\
Chemotherapy in visceral crisis disease & 23 & $65.72 \%$ \\
First line of therapy & 23 & $65.72 \%$ \\
Treatment modality & \\
$\quad$ Epirubicine and cyclophosphamide & 9 & $25 \%$ \\
$\quad$ Paclitaxel and bevacizumab & 7 & $20 \%$ \\
$\quad$ Docetaxel & 7 & $20 \%$ \\
\hline Mode of administration IV & 23 & $65.72 \%$ \\
Number of cycle of chemotherapy infusion & 21 & $91.30 \%$ \\
$\quad$ One cycle & 2 & $8.70 \%$ \\
$\quad$ Two cycle & & $4.7 \pm 1.9$ weeks \\
The mean time between visceral crisis and death & & \\
\hline
\end{tabular}

apy, worse ECOG PS score was associated with significantly shorter OS $(\mathrm{P}=0.001)$.

\section{Discussion}

Hormone receptor (HR) positive breast cancers have substantial variation. With advancements in the treatment of $\mathrm{HR}^{+} / \mathrm{HER}^{2-} \mathrm{mBC}$, it is important to identify the patients who will develop visceral crisis. The current study demonstrated that patients with visceral crisis had significantly worse outcomes as measured by short survival. On average, the death occurred in all patients within 4.7 weeks from visceral crisis diagnosis. Alternatively, little is known about serial events associated with visceral crisis. The change in tumor attributes could be a pure biological phenomenon, caused by acquisition of genetic changes either due to genomic instability of the tumour cells or because of selection due to endocrine treatment. In our cohort, the visceral crisis was distributed as follows: carcinomatous meningitis or leptomeningeal metastases in $20 \%$, significant liver metastases causing liver functional compromise - hepatocellular failure - raised bilirubin in absence of extrahepatic obstruction, elevated transaminases in $55 \%$, lymphangitic lung metastasis, or bulky lung metastases causes' respiratory failure in $35 \%$. Repeat biopsy of metastatic lesions was not performed in our patients. Considering that biopsy should be avoided when it is not safe and when the results are not expected to lead to any changes in treatment. In real-world practice, patients with visceral crisis are more likely to receive chemotherapy, often because their vital organs are affected [3]. The current study showed that different regimenbased chemotherapies have limited efficacy and effectiveness. Despite the fact that visceral crisis was cited as the primary cause of death in all patients receiving chemotherapy, $65 \%$ of our patients receiving chemotherapy underlined the difficulty of discussions in patients from Moroccan societies. Oncologists are not always aware or accepting a patient with visceral crisis, and they may be motivated to continue to treat the patient by a strong desire to help them, despite survival chances. Furthermore, the study showed that two-thirds of patients went to the emergency department or were admitted to the hospital at their visceral crisis, suggesting that patients may also play an active role in the high rate of cytotoxic treatment at visceral crisis. Chemotherapy in visceral crisis disease confers no survival advantage when compared to supportive care (5.8 weeks vs. 6.2 weeks, $P=0.23$ ), a study has found. Despite all limitations of the small patient cohort and the retrospective nature of this study. Conventional endocrinetherapy combined to novel targeted therapies (CDK-4/6 inhibitors and mTOR inhibitor) for the treatment of $\mathrm{HR}^{+} / \mathrm{HER}^{2-} \mathrm{mBC}$ has not been performed in our patient population. However, the current data report interesting insight into a very complex palliative cohort of visceral crisis in luminal $\mathrm{mBC}$. Furthermore, this observational retrospective study shows gruelling treatments given to people with visceral crisis illnesses have no clinical benefit. Instead, it can actually lead to a worse quality of life. Our study found that $25 \%$ of patients and $65 \%$ died respectively in the hospital and at home. This is true in the context of a country with little availability of palliative care resources, where health policies should be more focused on incorporating palliative medicine in all medical strategies. Efforts are critically needed to improve care for this patient population to ensure that the care provided meets the goals and preferences of patients. In summary, our data showed that management of luminal $\mathrm{mBC}$ with visceral crisis is a complex problem. Prognosis is dismal as measured by short survival. Chemotherapy has limited efficacy and effectiveness. This study could provide important evidence for medical decision, such as shedding light on the optimal sequencing of endocrine therapy, chemotherapy and identifying the patients who need chemotherapy followed by maintenance endocrine therapy. We need prospective multicentric studies, with testing tools allowing better sharing of the decisions on active treatment between the medical team, the patient and his family. 


\section{Acknowledgments}

We would like to thank the medical records section of Mohamed V Teaching Military Hospital who facilitated data collection for conduction of the present study.

\section{Disclosure}

All data generated or analyzed during this study are included in this published article. The authors presented all the necessary information about their case report in the manuscript. However, about the literature review, all used literature was referenced appropriately in the "references" section.

\section{Conflicts of Interest}

The authors declare that they have no competing interests.

\section{Funding}

The authors disclose that they have no financial relationships.

\section{Author Contributions}

SY conceived the original idea for the study design and drafted the manuscript. $\mathrm{KS}, \mathrm{AD}, \mathrm{AM}, \mathrm{KH}$ and $\mathrm{LA}$ participated in data collection and revised the manuscript. MI and HE coordinated the study and gave critical comments on the draft manuscript. All authors read and approved the final manuscript.

\section{Abbreviations}

SD: standard deviation; OS: overall survival; CBC: complete blood count; CA: cancer antigen; CT: computed tomography; ER: estrogen receptor; PgR: progesterone receptor; HER2: human epidermal growth factor 2; CDK: cyclin-dependent kinase; mTOR: mammalian target of rapamycin

\section{References}

1. Sandoval C, Rahal R, Forte T, Klein-Geltink J, He D, Bryant $\mathrm{H}$. Indicator measures $\mathrm{er} / \mathrm{pr}$ and her2 testing among women with invasive breast cancer. Curr Oncol. 2013;20(1):62-63.
2. Wolff AC, Hammond ME, Schwartz JN, Hagerty KL, Allred DC, Cote RJ, Dowsett M, et al. American Society of Clinical Oncology/College of American Pathologists guideline recommendations for human epidermal growth factor receptor 2 testing in breast cancer. J Clin Oncol. 2007;25(1):118-145.

3. Cardoso F, Costa A, Norton L, Senkus E, Aapro M, Andre F, Barrios $\mathrm{CH}$, et al. ESO-ESMO 2nd international consensus guidelines for advanced breast cancer $(\mathrm{ABC} 2)$ dagger. Ann Oncol. 2014;25(10):1871-1888.

4. Partridge AH, Rumble RB, Carey LA, Come SE, Davidson NE, Di Leo A, Gralow J, et al. Chemotherapy and targeted therapy for women with human epidermal growth factor receptor 2-negative (or unknown) advanced breast cancer: American Society of Clinical Oncology Clinical Practice Guideline. J Clin Oncol. 2014;32(29):33073329.

5. Gavila J, Lopez-Tarruella S, Saura C, Munoz M, Oliveira M, De la Cruz-Merino L, Morales S, et al. SEOM clinical guidelines in metastatic breast cancer 2015. Clin Transl Oncol. 2015;17(12):946-955.

6. Guia GEICAM de PracticaClinica Para el Diagnostico y Tratamientodel Cancer de Mama Metastasico. 2015.

7. Beslija S, Bonneterre J, Burstein HJ, Cocquyt V, Gnant M, Heinemann V, Jassem J, et al. Third consensus on medical treatment of metastatic breast cancer. Ann Oncol. 2009;20(11):1771-1785.

8. Cruz Jurado J, Richart Aznar P, Garcia Mata J, Fernandez Martinez R, Pelaez Fernandez I, Sampedro Gimeno T, Galve Calvo E, et al. Management of patients with metastatic breast cancer. Adv Ther. 2011;28(Suppl 6):50-65.

9. Yardley DA, Noguchi S, Pritchard KI, Burris HA, 3rd, Baselga J, Gnant M, Hortobagyi GN, et al. Everolimus plus exemestane in postmenopausal patients with $\mathrm{HR}(+)$ breast cancer: BOLERO-2 final progression-free survival analysis. Adv Ther. 2013;30(10):870-884.

10. Baselga J, Campone M, Piccart M, Burris HA, 3rd, Rugo HS, Sahmoud T, Noguchi S, et al. Everolimus in postmenopausal hormone-receptor-positive advanced breast cancer. N Engl J Med. 2012;366(6):520-529.

11. Finn RS, Crown JP, Lang I, Boer K, Bondarenko IM, Kulyk SO, Ettl J, et al. The cyclin-dependent kinase 4/6 inhibitor palbociclib in combination with letrozole versus letrozole alone as first-line treatment of oestrogen receptor-positive, HER2-negative, advanced breast cancer (PALOMA-1/TRIO-18): a randomised phase 2 study. Lancet Oncol. 2015;16(1):25-35.

12. Hortobagyi GN, Stemmer SM, Burris HA, Yap YS, Sonke GS, Paluch-Shimon S, Campone M, et al. Ribociclib as first-line therapy for hr-positive, advanced breast cancer. N Engl J Med. 2016;375(18):1738-1748. 